\title{
MÉTODOS ESTADÍSTICOS UTILIZADOS EN LAS CIENCIAS DEL MOVIMIENTO HUMANO
}

\author{
José Moncada Jiménez \\ Profesor en la Escuela de Educación Física y Deportes
}

\author{
Recibido 29-X-2003 • Aceptado 9-III-2004
}

"Con un poco de habilidad matemática, se puede hacer pasar seudociencia por ciencia auténtica"

M. Bunge

\begin{abstract}
Resumen: El propósito de este estudio fue describir los métodos estadísticos más utilizados en una revista del área de las ciencias del movimiento humano indexada en el Science Citation Index, la cual obtuvo en el año 1999 un factor de impacto 2.110. De los 305 artículos publicados durante el año 2001, se encontró que la mayoría $(45,2 \%)$ provenían de los Estados Unidos de América, principalmente en el área de la fisiología aplicada al deporte o al ejercicio (65,9\%). Las técnicas de análisis estadístico más usadas fueron las de tipo paramétrico (72\%): análisis de varianza en sus diversos modelos (57,7\%), $t$ student $(34,8 \%)$, correlación (29,5\%) y análisis de regresión (21\%). Las técnicas de análisis no paramétrico $(12,8 \%)$ más utilizadas fueron: chi cuadrado (7,9\%) y Wilcoxon (4,9\%). En el 12,8\% de los estudios se reportaba el uso combinado de técnicas paramétricas y no paramétricas. También se realizaron pruebas de seguimiento (post hoc), y otras para analizar supuestos teóricos en los que se basan las pruebas paramétricas. El 61,6\% de los investigadores no reportaron haber usado un paquete estadístico para analizar sus datos; mientras que aquellos que sí lo hicieron (38,4\%), reportaron haber usado el programa Statistical Package for the Social Sciences (13,4\%). En resumen, se observó que la mayoría de los estudios publicados en una revista científica de alto índice de impacto, que a su vez refleja la
\end{abstract}

\section{Introducción}

Actualmente se señala que el conocimiento científico, derivado de los grandes esfuerzos que realizan los investigadores en las diversas áreas del saber humano, llámense ciencias sociales, ciencias básicas y biológicas, puede ser consultado, analizado, criticado, y utilizado o descartado por la comunidad científica. Aunque hoy en día existen diferentes formas para consultar dicha información, se reconoce que la publicación científica, específicamente el artículo de investigación impreso en una revista, es considerada la manera "tradicional” más utilizada entre los investigadores para la diseminar sus hallazgos (Giménez Toledo, Gómez Caridad, Martín Sempere, Páez Mañá, Román Román, Urdín Caminos, y Vázquez Valero, 2001).

En el proceso inevitable de someter un trabajo a la revisión crítica de otros, lo 
calidad de los manuscritos, fueron elaborados tomando en consideración un apropiado diseño y el uso adecuado de técnicas estadísticas avanzadas. Se sugiere a los educadores físicos, científicos del movimiento humano, readecuar los cursos de metodología de la investigación y análisis estadísticos para que incluyan las técnicas descritas en este documento. Palabras claves: métodos de investigación, estadistica, bibliometría, ciencias del movimiento humano.

Palabras clave: Métodos de búsqueda, Bibliometría, Estadística, Movimiento Humano, Ciencias.

\begin{abstract}
The purpose of this study is to describe the most cited statistical methods reported in a scientific journal of the human movement sciences area. The journal was indexed in the Science Citation Index, and was rated in 1999 with an impact factor $=2.110$. Most of the 305 articles published in 2001 belonged to United States researchers (45,2\%), with a clear concentration in exercise and/or sports physiology (65,9\%). The most common statistical analysis performed (72\%) were the following parametric techniques: analysis of variance and its different models $(57,7 \%)$, student $t$-tests $(34,8 \%)$, correlation $(29,5 \%)$, and regression analysis (21\%). Non parametric techniques $(12,8 \%)$ reported were: chi squared $(7,9 \%)$ and Wolcoxon (4,9\%). Also, 12,8\% of the studies showed a combined use of parametric and non parametric techniques in their data analysis. Follow-up tests (post hoc), as well as other assumption analysis were reported. Researchers $(61,6 \%)$ did not report using a statistical or computerized package to analyze their data. From those who did use a package (38,4\%), $13,4 \%$ preferred to use the Statistical Package for the Social Sciences to analyze their data. In summary, most studies published in a high-quality journal were properly designed and data analyzed, relying on advanced statistical analysis. Physical educators and movement sciences specialists are suggested to modify their research methods and statistical analysis courses in order to provide a better understanding of the statistical techniques reported in this document. Key words: research methods, bibliometrics, statistics, human movement sciences.
\end{abstract}

Key words: Research Methods, Bibliometrics, Statistics, Human Movement, Sciences. que se conoce en el ámbito académico científico como la "revisión de pares" o el "arbitraje de pares", se requiere que el investigador posea no sólo la madurez para aceptar críticas ajenas, sino también, la seguridad y confianza de que el trabajo que envía para consideración de un consejo editorial es sólido, sustentable, y que aporta nuevo conocimiento (International Council for Science, 1999; Thomas y Nelson, 2001; Salazar Rojas, 2002).

La Asociación Americana de Psicología (APA) menciona que existen diversos criterios de forma y de fondo que se utilizan para evaluar un estudio sometido a consideración para publicación en una revista; criterios con los que al final de cuentas se debe decidir si se acepta o se rechaza el trabajo (APA, 1994). Entre ellos se pueden mencionar aspectos tales como el contenido y estilo de la escritura (e.g., extensión de la información presentada, uso efectivo de la información, organización y claridad de la revisión, análisis y síntesis de la información, y profundidad y calidad de la documentación), la mecánica de la escritura en sí (e.g., estilo técnico de escritura impersonal, puntuación, tiempo de las oraciones, gramática, ortografía, y estructura de los párrafos), y por supuesto, la bibliografía (e.g., orden alfabético o numérico, formato de acuerdo con guías nacionales o internacionales, uso de fuentes primarias y secundarias).

Sin embargo, de acuerdo con Baumgartner y Strong (1998), los siguientes aspectos también deberían tomarse en cuenta seriamente a la hora de evaluar un artículo sometido a consideración para publicación: a) la originalidad del estudio; b) la profundidad y exhaustividad de la revisión bibliográfica (marco teórico); c) el diseño del estudio; d) el análisis de los datos; e) la presentación y coherencia de los resultados; y f) las conclusiones lógicas y nuevos aportes derivados de los resultados presentados.

Aunque todos los aspectos listados anteriormente merecen un trato detallado, 
a continuación se desea estudiar solamente uno de ellos, el análisis de los datos. Para ello, se ha seleccionado intencionalmente, la que puede considerarse como la tercera revista científica más importante en las ciencias del movimiento humano, Medicine \& Science in Sports \& Exercise ${ }^{\circledR}$ (MSSE®) (ISSN: 0195-9131). Esta revista es el medio de divulgación científica oficial del American College of Sports Medicine (ACSM), institución creada en 1954, y considerada como la organización más importante del mundo en lo que a ciencias del movimiento humano y la medicina deportiva se refiere.

La revista MSSE $\AA$, cuyo nombre se podría traducir como Medicina y Ciencia en el Deporte y el Ejercicio, pertenece a la compañía estadounidense Wolters Kluwer Company, y es impresa y distribuida por la casa editorial Lippincott Williams \& Wilkins. MSSE® presentó un factor de impacto (FI) de 2.110 en el año 1999, ligeramente menor al FI $=2.327$ de la revista American Journal of Sports Sciences®, y mucho menor que la revista más importante en el área de la actividad física, el deporte y la recreación, Exercise Immuno$\log y$ Review ${ }^{\circledR}(\mathrm{IF}=2.900)$ (Thomas $\mathrm{y}$ Nelson, 2001). El FI, obtenido del Science Citation Index (SCI), es un parámetro bibliométrico utilizado para juzgar la calidad de una revista, y se calcula como el número de veces promedio que los artículos publicados en la revista son citados durante un año. Este indicador ha sido criticado por varios autores (Gibbs, 1995) y es tema polémico actualmente; sin embargo, es uno de los indicadores más conocidos y aceptados internacionalmente para evaluar la calidad de una publicación científica (Lomonte y Ainsworth, 2002).

Por lo tanto, en el presente trabajo se describen algunas de las características de los estudios publicados durante todo un año en una revista científica con consejo editorial y revisión de pares, poniendo especial énfasis a las técnicas de análisis estadístico.

\section{Metodología}

\section{Selección del material}

Se seleccionó intencionalmente el volumen № 33 de la revista MSSE®, el cual estaba compuesto de 12 números (un número mensual). Se excluyó del análisis el suplemento del mes de mayo del año 2001, que corresponde a la memoria del congreso anual del ACSM, la cual solamente contenía resúmenes de ponencias. En total, se analizaron 305 artículos.

\section{Procedimientos}

Se tomó cada revista y se buscó y codificó la siguiente información. Primero, el mes de publicación del artículo. Segundo, se obtuvo el nombre del país en donde se realizó la investigación o el país de procedencia del primer autor del estudio para explorar la productividad de un país o región. Tercero, se codificó el área de estudio, para lo cual se definieron las categorías siguientes: a) fisiología del ejercicio o del deporte; b) biomecánica; c) epidemiología de la actividad física; d) nutrición deportiva; y e) psicología o psicofisiología. Posteriormente se obtuvo el tipo de análisis estadístico, categorizado de la siguiente forma: a) paramétrico; b) no paramétrico; y c) combinación de paramétrico y no paramétrico. Luego se obtuvo el nombre del programa o paquete estadístico utilizado para analizar los datos. Finalmente, se obtuvo el nombre de la técnica estadística utilizada para analizar los datos.

\section{Análisis estadístico}

La información fue codificada y vaciada en el Statistical Package for the Social Sciences (SPSS $®)$, con el que se obtuvieron las estadísticas descriptivas (\%) necesarias. 


\section{Resultados}

El desglose de los artículos analizados ( $\mathrm{n}=305$ ) por número de revista se presenta en la tabla 1. Como puede observarse, la cantidad de artículos publicados por número varía de 24 a 27. Del total de artículos, 45,2\% fueron publicados por investigadores de los Estados Unidos de América (USA), Canadá (7,9\%), Australia (7,9\%), Inglaterra $(6,6 \%)$, y otros 24 países.

Tabla 1

Desglose de los artículos del volumen 33 de MSSE®.

\begin{tabular}{lccc}
\hline $\begin{array}{l}\text { Mes } \\
\text { (número) }\end{array}$ & $\begin{array}{c}\text { Número } \\
\text { de artículos }\end{array}$ & $\%$ & $\begin{array}{c}\% \\
\text { acumulado }\end{array}$ \\
\hline Enero (1) & 25 & 8,2 & 8,2 \\
Febrero (2) & 26 & 8,5 & 16,7 \\
Marzo (3) & 25 & 8,2 & 24,9 \\
Abril (4) & 26 & 8,5 & 33,4 \\
Mayo (5) & 25 & 8,2 & 41,6 \\
Junio (6) & 27 & 8,9 & 50.5 \\
Julio (7) & 25 & 8,2 & 58,7 \\
Agosto (8) & 25 & 8,2 & 66,9 \\
Setiembre (9) & 26 & 8,5 & 75,4 \\
Octubre (10) & 26 & 8,5 & 83,9 \\
Noviembre (11) & 25 & 8,2 & 92,1 \\
Diciembre (12) & 24 & 7,9 & 100,0 \\
\hline Total & 305 & 100,0 & \\
\hline
\end{tabular}

El área de la fisiología aplicada al ejercicio o al deporte fue la que produjo mayor cantidad de estudios $(65,9 \%)$, seguida por el área de biomecánica $(13,4 \%)$, epidemiología de la actividad física $(10,8 \%)$, nutrición deportiva $(8,2 \%)$, y finalmente, la psicología del ejercicio o psicofisiología $(1,6 \%)$.

Las técnicas de análisis estadístico reportadas fueron las de tipo paramétrico (72\%), no paramétricas $(5,6 \%)$, y la combinación de las dos anteriores (12,8\%). En 7 estudios $(2,3 \%)$, únicamente se reportaron estadísticas descriptivas (e.g., promedios, desviación estándar, \%), y hubo 22 revisiones de literatura $(7,2 \%)$, en las cuales no se utilizaron técnicas estadísticas.
En cuanto al uso de paquetes o programas computacionales utilizados como herramientas para el análisis estadístico, se encontró que $61,6 \%$ de las investigaciones publicadas no reportaron el uso de éstos; mientras que en el 38,4\% de los casos sí se reportó el uso de ayudas computacionales. Se indicó que el programa SPSS® fue el más utilizado $(13,4 \%)$, seguido del SAS ó SAS-JMP $®(8,5 \%)$, Statistica ${ }^{\circledR}(3,3 \%)$, StatView ${ }^{\circledR}(2,6 \%)$, nQuery Advisor ${ }^{\circledR}(2,0 \%)$, la combinación de varios paquetes $(1,6 \%)$, Datadesk® $(1,0 \%)$, SYSTAT ${ }^{\circledR}(1,0 \%)$, y otros con porcentajes menores al $1,0 \%$.

Las técnicas de análisis estadístico más utilizadas se presentan en la tabla 2 . Se observa que entre las técnicas de análisis paramétrico sobresale el análisis de varianza (ANOVA). Un desglose más minucioso indicó que las técnicas de ANOVA utilizadas fueron de una vía $(25,6 \%)$, dos vías o mixta $(20,7 \%)$, tres vías $(3,0 \%)$, o combinaciones de las anteriores $(8,2 \%)$.

Cuando el ANOVA arrojaba resultados estadísticamente significativos, los investigadores procedían a realizar un análisis de seguimiento (i.e., efectos principales, efectos simples, post hoc). El análisis post hoc consiste básicamente en la realización de comparaciones múltiples de promedios (Hyllegard, Mood, y Morrow, 1996; Yaremko, Harari, Harrison, y Lynn, 1982). Los análisis post hoc más utilizados fueron el de Tukey ó Tukey HSD (10,8\%), Scheffè, Bonferroni, y Newman-Keuls $(5,2 \%$ cada uno, respectivamente). También se utilizaron el de Fisher LSD (4,3\%), Duncan $(1,6 \%)$, Dunnet $(1,3 \%)$, Least Squares Means $(0,3 \%)$, y contrastes lineales $(0,3 \%)$.

Las pruebas t-student también fueron utilizadas $(34,8 \%)$ en los artículos publicados en el volumen 33 de MSSE®. La prueba t-student para observaciones pareadas o dependientes fue la más utilizada $(16,7 \%)$, seguida de la prueba t-student para grupos u observaciones independientes $(14,1 \%)$, y finalmente, la prueba t-student para una muestra (one-sample t-test) 
Tabla 2

Resumen de las técnicas de análisis estadístico más utilizadas en el volumen 33 de MSSE®.

\begin{tabular}{llll}
\hline \multicolumn{1}{c}{ Tipo de Análisis Estadístico } & \\
\hline \multicolumn{1}{c}{ Paramétrico } & $\%^{*}$ & No Paramétrico & $\%^{*}$ \\
\hline Análisis de Varianza (ANOVA) & 57,7 & Chi cuadrado ( $\left.\chi^{2}\right)$ & 7,9 \\
t-student & 34,8 & Prueba de Wilcoxon & 4,9 \\
Correlación producto momento de Pearson & 29,5 & Prueba U de Mann-Whitney & 3,0 \\
Análisis de regresión & 21,0 & ANOVA de Kruskall-Wallis & 1,6 \\
Análisis de covarianza (ANCOVA) & 5,2 & ANOVA de Friedman & 1,3 \\
Análisis multivariado de varianza (MANOVA) & 3,0 & Prueba de Mantel-Haenszel & 1,0 \\
Correlación canónica & 0,3 & Prueba de exactitud de Fisher & 0,3 \\
\hline
\end{tabular}

Nota: *: \% de veces utilizado del total de artículos publicados.

$(0,7 \%)$. Se reporta también el uso de combinaciones de estas pruebas $(3,3 \%)$.

Los análisis basados en correlación también fueron empleados en los estudios publicados. Se observó que la técnica de correlación bivariada más usada fue la producto momento de Pearson (29,5\%). Por otra parte, se aplicaron técnicas de análisis de regresión (21\%), de tipo lineal simple $(5,2 \%)$, múltiple $(11,8 \%)$, y logística $(2,6 \%)$; aunque también se utilizaron combinaciones de éstas (1,3\%). Finalmente, las tres técnicas paramétricas menos reportadas fueron el análisis de covarianza, el análisis multivariado de varianza, y la correlación canónica (Tabla 2).

Respecto a las técnicas de análisis no paramétrico, en general se observa una reducida utilización. Sin embargo, se destaca el uso de la prueba no paramétrica por excelencia, Chi cuadrado $\left(\chi^{2}\right)$ $(7,9 \%)$.

Finalmente, los investigadores calcularon estadísticos muy específicos, como por ejemplo los riesgos relativos (RR), los cuales son ampliamente usados en investigaciones epidemiológicas. También se reportaron técnicas para estimar el tamaño de la muestra (i.e., n) y la potencia, se calcularon coeficientes de variación, porcentajes de cambio $(\Delta \%)$, tamaño del efecto o varianza compartida (e.g., $\varepsilon^{2}, \omega^{2}, R^{2}$ ), pruebas para determinar la homogeneidad de las varianzas (e.g., Bartlett, D’Agostino, Ryan-Joiner, Kolmogorov-Smirnov, Levene), pruebas para estimar la normalidad de la distribución de los puntajes (e.g., Shapiro-Wilk), se realizaron trasformaciones logarítmicas y ajustes para no violar el supuesto de esfericidad (e.g., Huyn-Feldtepsilon), se calculó una correlación punto biserial, se estudió la autocorrelación en los modelos de regresión lineal múltiple con el estadístico Durbin-Watson, se llevó a cabo un análisis post hoc de Tamhane, una regresión Cox y Poisson, pruebas de confiabilidad, y finalmente, se utilizó el gráfico Bland-Altman para estudiar la concordancia entre dos métodos propuestos por un investigador.

\section{Discusión}

El número de artículos publicados refleja la consistencia en la estructura de la revista y en la cantidad y calidad de la información recibida. La producción científica internacional se ve reflejada en el porcentaje de investigaciones publicadas en la revista por investigadores de USA o por extranjeros que realizaron las investigaciones en USA como parte de sus pasantías, estudios 
de posgrado o convenios de investigación. Los porcentajes observados en este estudio concuerdan con los presentados por la Organización de las Naciones Unidas para la Educación, la Ciencia y la Cultura (UNES$\mathrm{CO}, 2001$ ), en donde se afirma que los países de América del Norte (USA y Canadá) son los responsables de la mayor cantidad de publicaciones científicas en el mundo $(36,6 \%)$, sólo después de sumar las publicaciones de todos los países europeos juntos $(37,5 \%)$.

Es meritorio mencionar que varios investigadores brasileños fueron los únicos latinoamericanos que pudieron publicar dos trabajos en la revista, los cuales representan el 0,7\% del total de investigaciones publicadas ese año. Tal vez alguien podría argumentar que no tener el idioma inglés como lengua materna podría ser el principal obstáculo para no poder publicar en MSSE®; los cual es absolutamente cierto; sin embargo, investigadores españoles y franceses, por ejemplo, también aportaron sus investigaciones $(2,3 \%$ y $5,2 \%$, respectivamente).

Las áreas de estudio de mayor representatividad expuestas en la revista MSSE® incluyen la fisiología, en sus dos grandes áreas, la deportiva y la del ejercicio. La fisiología deportiva se puede entender como el estudio de los sistemas orgánicos en el atleta, o visto de otra manera, como las respuestas y adaptaciones del organismo en los deportistas. Por otro lado, la fisiología del ejercicio se podría comprender como el estudio de los cambios agudos y de las adaptaciones crónicas en el organismo de las personas físicamente activas, quienes no necesariamente son atletas. Es evidente la contundente proporción de estudios en el área de la fisiología, lo que refleja el enfoque biológico de la revista. Es evidente que la baja proporción de artículos en otras áreas obedece a que existen otras revistas especializadas donde los investigadores envían sus trabajos. Por lo tanto, la cantidad de artículos publicados en esas áreas en esta revista específica subestiman la producción total de la investigación que se realiza en otras áreas.

De los artículos publicados, se nota la cantidad abrumadora de estudios en los que se utilizan análisis de datos que incluyen técnicas estadísticas paramétricas o la combinación de técnicas paramétricas y no paramétricas. Se observa la escasa cantidad de estudios publicados en los que únicamente se utilizan técnicas estadísticas no paramétricas o simplemente estadísticas descriptivas. No se desea menospreciar de ninguna forma el uso de estas dos últimas, ya que son absolutamente necesarias para resolver ciertas situaciones en las que no es apropiado utilizar técnicas paramétricas debido, por ejemplo, a la violación del supuesto de normalidad de las distribuciones de puntajes.

Los paquetes o programas estadísticos se han diversificado a través de los años. Por ejemplo, el SPSS $®$, cuyas siglas se refieren inicialmente a las ciencias sociales, ha extendido su uso por ejemplo, a los campos de la fisiología y la biomecánica. En el análisis de este trabajo se observó que a más de la mitad de los investigadores no les interesa mencionar el paquete estadístico que utilizaron para analizar los datos (asumiendo que no hicieron los análisis con calculadora), lo que denota por una parte imparcialidad para endorsar o promover el uso de una determinada marca o producto, y por otro lado, las fortalezas y debilidades en el uso de uno u otro paquete estadístico para análisis de experimentos. Por otra parte, se nota una clara tendencia a que las grandes compañías que desarrollan programas de computación también ofrecen diferentes marcas. Por ejemplo, la compañía SPSS ofrece los paquetes estadísticos SPSS $®$, SigmaPlot $\AA$ y SigmaStat $\AA$, que juntos representan $14,5 \%$ de los paquetes reportados en el presente análisis. Por su lado, la compañía SAS Institute ofrece los paquetes SAS®, StatView® y JMP®, que en conjunto 
representan un $11,1 \%$. En todo caso, queda un debate abierto para defender la posición con respecto al uso de un paquete estadístico determinado.

De las técnicas de análisis estadístico utilizadas en las investigaciones publicadas en el volumen 33 de MSSE®, se nota que el ANOVA, en sus distintas modalidades, fue la técnica más seleccionada. La prueba de ANOVA es considerada como "robusta" (Kerlinger, 1988; Kerlinger y Lee, 2001), indicando que la violación de los supuestos de normalidad en la distribución de los datos no afecta gravemente el cálculo final de la razón $\mathrm{F}$, y por lo tanto, la probabilidad de cometer un error de tipo I. Cuando la razón $F$ de la prueba de ANOVA era significativa, se realizaba el análisis de seguimiento apropiado. Así, se utilizaron análisis post hoc conservadores (e.g., Bonferroni) y otros más liberales (e.g., Fisher LSD) (Yaremko et al., 1982).

Se observa que las pruebas t-student también fueron utilizadas, especialmente en diseños de medidas repetidas; es decir, en diseños en los cuales el mismo sujeto era medido en la variable dependiente en dos ocasiones. Lo anterior indica una leve preferencia por diseños de investigación fuertes, en los que el mismo sujeto es su propio control, en contraposición a los diseños en que se estudia la variabilidad entre distintos grupos de sujetos (i.e., grupos independientes) (Ary, Jacobs, y Razavieh, 1989; Kerlinger, 1988).

Las técnicas basadas en correlación también empiezan a ser más utilizadas, especialmente los modelos de predicción, los cuales utilizan el análisis de regresión como su herramienta de análisis principal. Por ello, parece que las técnicas de regresión comienzan a usarse cada día más y se espera que aumente su uso debido a que hoy en día es mucho más fácil realizar los análisis con la ayuda de una computadora. Por su parte, la técnica de análisis de regresión logística, es una herramienta útil debido a que se pueden combinar variables categóricas y continuas para predecir la ocurrencia o no de un evento. Esto difiere al uso del análisis de regresión lineal múltiple, en el que dos o más variables (predictoras) medidas en una escala continua predicen el valor de una variable (criterio), también medida en una escala continua (Kerlinger, 1988).

Respecto al uso de las pruebas estadísticas no paramétricas, se destacan las análogas a las pruebas t-student y ANOVA paramétricas. Pero lo más sobresaliente es el uso de la prueba de Chi cuadrado $\left(\chi^{2}\right)$. Las pruebas no paramétricas han sido recomendadas cuando los supuestos de normalidad de la distribución de puntajes y la homogeneidad de la varianza han sido violados (Thomas y Nelson, 2001; Thomas, Nelson, y Thomas, 1999).

En Costa Rica, y específicamente en el área de las ciencias del movimiento humano, por múltiples razones tradicionalmente no se han utilizado técnicas de análisis estadístico no paramétricas y se ha dejado de lado la enseñanza de técnicas paramétricas más avanzadas (e.g., correlación canónica, MANOVA, regresión lineal múltiple); sin embargo, se podrían comenzar a enseñar algunas de estas técnicas con la ayuda de excelentes publicaciones realizadas al respecto, como la de Ang (1998) acerca de correlación canónica y la de Thomas et al. (1999), acerca de análisis no paramétricos.

Por último, se utilizaron, aunque en una pequeña proporción, una serie de pruebas estadísticas que sirven para estudiar los supuestos teóricos en los que se basan las pruebas paramétricas. Por ejemplo, se evaluaron los supuestos de normalidad de la distribución de los puntajes y la homogeneidad de las varianzas. Por otra parte, se realizaron cálculos para estimar el tamaño de la muestra, y algo sumamente importante, brindar una aproximación a la cantidad de varianza compartida (e.g., $\mathrm{R}^{2}$ ) o explicada $\left(\right.$ e.g., $\left.\omega^{2}\right)$. De acuerdo con Thomas et al. (1999), estos tipos de pruebas estadísticas 
deberían ser reportadas por los investigadores cuando desean que sus artículos sean tomados con mayor seriedad por un consejo editorial, especialmente porque muchas de las variables que se estudian en los diversos campos de las ciencias del movimiento humano no presentan distribuciones normales, por ejemplo, datos provenientes de variables de laboratorio clínico o de mediciones psicométricas.

En resumen, las técnicas estadísticas que un investigador que desea publicar en una revista científica como MSSE® y que debería conocer, se basan en el análisis de la varianza, la correlación y la predicción. El conocimiento de las técnicas estadísticas no garantizan que un trabajo sometido a consideración para su publicación sea aceptado; sin embargo, es un respaldo que asegura un adecuado análisis de la información y conclusiones basadas en evidencia objetivamente analizada. No cabe la menor duda de que con la inserción de las computadoras en el ámbito investigativo, ahora es posible utilizar técnicas de análisis multivariado que hace muchos años era casi imposible calcular. Por ello, en las universidades se deberá preparar a los futuros investigadores, científicos del movimiento humano, para que no solo conozcan, comprendan, apliquen y analicen técnicas básicas de ANOVA y regresión; sino que también conozcan los conceptos fundamentales en los que se basan las técnicas de análisis multivariado más avanzadas. También, desde el punto de vista curricular, parece que se debe comenzar a introducir más cursos de análisis multivariado y técnicas de análisis no paramétrico en las ciencias del movimiento humano, y no dejar esos cursos solamente para estudiantes de posgrado.

\section{Notas}

1. Los nombres que aparecen con el superíndice® son marcas comerciales debidamente registradas por compañías privadas. El autor de este artículo no tiene afiliación alguna a dichas compañías.

2. Se agradece a la Licda. Yamileth Chacón Araya y a la M. Sc. Maureen Meneses Montero por la revisión y comentarios durante la elaboración de este manuscrito.

\section{Referencias bibliográficas}

American College of Sports Medicine (Ed.). "Medicine and Science in Sports and Exercise". Vol. 33, Números 1 al 12. 2001.

American Psychological Association. Publication manual of the American Psychological Association (4 ${ }^{\text {th }}$ Ed.). Hyattsville, MD: American Psychological Association. 1994.

Ang, R. P. "Canonical correlation analysis as a general linear model: an effective instructional tool". Perceptual and Motor Skills. 87(3), 1315-1323. 1998.

Ary, D., Jacobs, L. Ch., y Razavieh, A. Introducción a la investigación pedagógica (2da. Ed.). México, DF: McGraw-Hill Interamericana de México. 1989.

Baumgartner, T. A. y Strong, C. H. Conducting and reading research in health and human performance (2 $\left.{ }^{\text {nd }} \mathrm{Ed}.\right)$. Boston, MA: WCB/McGraw-Hill. 1998.

Gibbs, W. W. "Lost science in the third world". Scientific American. 8, 9299. 1995.

Giménez Toledo, E., Gómez Caridad, I., Martín Sempere, M. J., Páez Mañá, J., Román Román, A., Urdín Caminos, C., y Vázquez Valero, M. La edición de revistas científicas: Guía de buenos usos. Madrid: Centro de Información y Documentación Científica CINDOC (CSIC). 2001. 
Hyllegard, R., Mood, D. P., y Morrow, J. R. Jr. Interpreting research in sport and exercise science. St. Louis, MO: Mosby-Year Book. 1996.

International Council for Science. Guidelines for Printing and Publishing. Paris: ICSU Press Committee on Dissemination of Scientific Information. 1999.

Kerlinger, F. N. Investigación del comportamiento (3ra ed.). México: McGraw-Hill Interamericana de México. 1998.

Kerlinger, F.N. y Lee, H.B. Investigación del comportamiento: métodos de investigación en ciencias sociales. México: McGraw-Hill. 2001.

Lomonte, B., y Ainsworth, S. "Publicaciones científicas de Costa Rica en el Science Citation Index: análisis bibliométricos del trienio 1999-2001". Revista de Biología Tropical. 50(3,4), 951-962. 2002.

Salazar-Rojas, W. "Principios del paradigma cuantitativo en la investiga- ción educativa”. Revista de Ciencias del Ejercicio y la Salud. 2(1), 61-71. 2002.

Thomas, J. R., y Nelson, J. K. Research methods in physical activity $\left(4^{\text {th }}\right.$ Ed.). Champaign, IL: Human Kinetics. 2001.

Thomas, J. R., Nelson, J. K., y Thomas, K. $\mathrm{T}$. "A generalized rank-order method for nonparametric analysis of data from exercise science: A tutorial". Research Quarterly for Exercise and Sport. 70(1), 11-23. 1999.

United Nations Organization for Education, Science and Culture (UNES$\mathrm{CO})$. The state of science and technology in the world. Montreal, Canada: The UNESCO Institute for Statistics. 2001.

Yaremko, R. M., Harari, H., Harrison, R. C., y Lynn, E. Reference handbook of research and statistical methods in psychology: for students and professionals. New York, NY: Harper \& Row. 1982. 